\title{
Psychometric Cognitive Decline Precedes the Advent of Subjective Cognitive Decline in the Evolution of Alzheimer's Disease
}

\author{
Barry Reisberg $^{a} \quad$ Yongzhao Shao $^{b} \quad$ Mesum Moosavi ${ }^{a}$ Sunnie Kenowsky ${ }^{a}$ \\ Alok Vedvyas ${ }^{c}$ Karyn Marsh $^{c}$ Jia Bao ${ }^{b}$ Maja Buj ${ }^{a}$ Carol Torossian ${ }^{a}$ \\ Alan Klugerc Gaurav Vedvyas ${ }^{c}$ Thet Oo ${ }^{c}$ Fawad Malik $^{\mathrm{e}}$ Fauzia Arain ${ }^{a}$ \\ Arjun V. Masurkarc Thomas Wisniewski ${ }^{\mathrm{a}, \mathrm{c}, \mathrm{d}}$ \\ ${ }^{a}$ Department of Psychiatry, NYU Langone Health, New York, NY, USA; ${ }^{b}$ Department of Population Health,

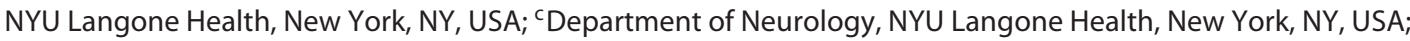 \\ ${ }^{\mathrm{d}}$ Department of Pathology, NYU Langone Health, New York, NY, USA; ${ }^{\mathrm{e}}$ Department of Pharmacy, \\ Bellevue Hospital, New York, NY, USA
}

\section{Keywords}

Psychometric cognition - Alzheimer's disease - Brain aging ·

Cognitive decline $\cdot$ Cognitive testing $\cdot$ Longitudinal studies

\begin{abstract}
Background: We have described the clinical stages of the brain aging and Alzheimer's disease (AD) continuum. In terms of the pre-dementia stages of $A D$, we introduced the terminology "mild cognitive impairment" $(\mathrm{MCl})$ for the first pre-dementia stage and "subjective cognitive decline" (SCD) for the pre-MCl stage. We now report the characteristics of a pre-SCD condition eventuating in likely AD. Objective: The aim of this study was to characterize a pre-SCD condition eventuating in AD. Method: Sixty healthy persons with "no cognitive decline" (NCD) were recruited and 47 were followed (mean baseline age, $64.1 \pm 8.9$ years; mean follow-up time, $6.7 \pm 3.1$ years). Outcome was determined at the final
\end{abstract}

assessment prior to 2002 as "decliner," if SCD or worse, or "nondecliner" if NCD. Results: After controlling for age, gender, years of education, and follow-up time, there was a between-group difference in the decline rate $(p<0.001)$. Also, after controlling for demographic variables and follow-up time, the combinatorial psychometric score was lower at baseline in the future decliners $(p=0.035)$. Of the 9 psychometric variables, after controlling for demographic variables and follow-up time, 3 were significantly lower at baseline in future decliners. Since AD is known to be age related and all subjects in this study were otherwise healthy, we also did an analysis without controlling for age. The combinatorial psychometric score was highly significantly better at baseline in the future nondecliners than in the future decliners $(p=$ 0.008). Conclusion: This is ostensibly the first study to link psychometric cognitive decline to the subsequent $S C D$ stage of eventual AD.

\section{KARGER}

(c) 2020 S. Karger AG, Basel

karger@karger.com

www.karger.com/dem
Barry Reisberg, MD

Department of Psychiatry, NYU Langone Health

145 East 32nd Street, Room 508

New York, NY 10016 (USA)

barry.reisberg@nyulangone.org 


\section{Introduction}

All of the research described below was conducted at the NYU Langone Medical Center and was approved by the NYU Langone Institutional Review Board. Written informed consent to participate in these studies was obtained from the subjects.

In 1982, on the basis of systematic clinical observations over the prior 4 years, we published the Global Deterioration Scale (GDS) which describes 7 major stages in the evolution of brain aging and the dementia of Alzheimer's disease (AD) [1]. The GDS identified a 3rd stage in which "the earliest clear-cut clinical deficits appear ... However objective evidence of memory deficit is obtained only through an intensive interview ..." [1]. In this GDS 3 stage, "co-workers become aware of the [person's] relatively poor performance" [1]. Also, "difficulties in finding words and names may become evident to intimates" [1]. In 1988, we coined the terminology "mild cognitive impairment" (MCI) for the 3rd GDS stage [2]. The GDS also identified a stage prior to MCI in which older persons have subjective complaints of cognitive decline only (SCD) [1]. This GDS stage 2 was described as a condition in which persons commonly complain of not recalling names and/or where they have placed familiar objects. These persons are otherwise healthy, and subsequent studies have shown that GDS stage 2 persons do not perform more poorly on psychometric tests than persons without these memory complaints [2].

In 1986, we hypothesized that SCD was a stage in otherwise healthy older persons and that the duration of this stage was 15 years prior to MCI [3]. In 2006, we published data which supported our temporal estimates [4] as described in an analysis in 2008 [5]. These analyses are summarized below.

Specifically, in our 2006 study [4], the nondecliners were followed over a mean of $8.9 \pm 1.8$ years. If GDS stage 2 is a stage lasting precisely 15 years, as hypothesized in Reisberg's 1986 publication [3], then $6.67 \%$ of subjects would be anticipated to decline annually over the 8.9-year mean interval of the 2006 study. Therefore, we would anticipate that $59.36 \%$ of subjects would decline to MCI or dementia over the 8.9-year mean study interval. The observed finding was that 27 of the 44 baseline subjects declined to MCI or dementia, i.e., $61.36 \%$ [5]. Hence, the observed decline rate differed from the hypothesized decline rate by only $2.00 \%$ over the 8.9 -year mean study interval, or $0.22 \%$ per year [5].

Recently, Mitchell et al. [6] conducted a meta-analysis of outcomes of persons with what they termed "subjective memory complaints" and obtained results remarkably congruent with our initial 1986 estimate of the duration of the SCD stage [3]. If the SCD stage lasts precisely 15 years, as estimated in 1986 [3], then we would expect $6.67 \%$ of a uniformly distributed population of SCD persons to move towards and convert to MCI per annum. In Mitchell et al.'s [6] analysis of 28 studies, there were 14,714 persons with subjective memory complaints (synonymous with SCD). Eleven of the studies could be analyzed in terms of the annual conversion rate (ACR) of developing MCI. They found that "from 11 studies the ACR of developing MCI was 6.67\%," a result precisely in accord with Reisberg's [3] 1986 initial temporal estimate of annual change in GDS stage 2 subjects.

With respect to our own studies, we believe that the remarkable precision of the above findings is the result in large part of the rigorous inclusion and exclusion criteria applied in our studies. Prior to inclusion, medical, psychiatric, neurologic, and neuroradiologic investigations are completed and subjects with concomitant conditions which might interfere with cognition, or which might progress or recur in a manner which might interfere with cognition, are excluded from our investigations. These exclusions result in robustly homogeneous subject groups.

In 2010, we published another study on the nature and outcome of persons in GDS stage 2, referred to in this 2010 investigation as "subjective cognitive impairment" [7]. This study directly compared outcomes of healthy subjects with and without SCD over a 7-year mean interval. Specifically, we compared outcomes of a consecutive series of subjects at GDS stage 1, i.e., with no subjective or objective declines, with those of subjects at GDS stage 2, i.e., with SCD only. Subject enrollment extended over a 14-year interval. Of 260 subjects comprising the baseline study population, follow-up was completed in 213 subjects (81.9\%), 47 with no baseline subjective or objective decline (GDS stage 1) and 166 with SCD only (GDS stage 2). Subject groups followed did not differ in the baseline age or Mini-Mental Status Examination (MMSE) scores. The follow-ups occurred over a mean of $6.8 \pm 3.4$ years. We observed that $14.9 \%$ of GDS stage 1 subjects who, by definition, were free of subjective complaints or objective evidence of cognitive decline at baseline, declined to a diagnosis of MCI or dementia. In contrast, $54.2 \%$ of GDS stage 2 subjects, by definition with subjective cognitive deficits at baseline, declined to MCI or dementia. After controlling for baseline demographic variables and follow-up time, a Weibull proportional hazards model revealed increased decline in the baseline GDS stage 2 sub- 
jects, with a hazard ratio of 4.5 , in comparison with the GDS stage 1 subjects. An accelerated failure time model analysis with an underlying Weibull survival function showed that GDS stage 2 subjects at baseline declined more rapidly, at $60 \%$ of the rate of the GDS stage 1 subjects. Furthermore, mean time to decline was 3.5 years longer for the GDS stage 1 than the GDS stage 2 subjects $(p=0.0003)$.

Largely as a result of the findings from our 7-year follow-up study, published in 2010, the scientific community's interest in the SCD entity greatly increased. This resulted in part in the creation of a Subjective Cognitive Decline Professional Interest Area (PIA) Working Group of the Alzheimer's Association. The PIA has sponsored 2 recent consensus publications on SCD, as well as other recent publications in which we have participated [8-11]. We now report that there is a stage of psychometric cognitive decline (PCD), which precedes the SCD stage in the evolution of the eventual overt dementia of AD.

\section{Methods}

For the present investigation, we selected subjects from the prior longitudinal investigation who were GDS stage 1 at baseline [7]. These GDS stage 1 persons were healthy and, by definition, free of either subjective or objective evidence of cognitive decline. Since our prior publication describes our selection criteria for the present investigation in detail, we also refer interested readers to our prior publication for this information [7].

\section{Subject Selection and Study Background}

Subjects were community residing, over 40 years of age, and recruited by public announcement or referral to participate in this longitudinal study on cognition and brain aging. The grant which provided support for this study from 1982 until 2003 was entitled: "Aging and Dementia: Longitudinal Course of Subgroups" (B. Reisberg, Principal Investigator). Additional support was provided by the New York University Alzheimer's Disease Center grant from 1990 to 2015 (S. Ferris, Principal Investigator, B. Reisberg, Director, Clinical Core, 1990-2014). The objective of these studies was to follow subjects at specified intervals, as long as possible, or until the subject's demise.

At baseline, medical, neurological, psychiatric, neuropsychological, clinical laboratory, and neuroradiologic evaluations were conducted to exclude subjects who, in terms of the present investigation, had conditions apart from normal brain aging, without SCD or MCI. Criteria for exclusion from the present investigation included (1) the presence of SCD, MCI, or dementia; (2) a history of clinically significant head trauma, seizures, mental retardation, or prior significant neurological disorder; (3) a modified Hachinski Ischemia Score of $\geq 4$ [12]; (4) a history of clinically significant cerebral infarction; (5) evidence of cerebral infarction from the brain neuroimaging evaluation (in the great majority of subjects, these were magnetic resonance imaging scans; in a small minority of subjects, these were computed tomography scans); (6) a signifi- cant history of drug or alcohol abuse; (7) a history of schizophrenia, major affective disorder, or the presence of a Hamilton Depression Scale [13] score of $\geq 16$; (8) the presence of cardiac, pulmonary, vascular, metabolic, or hematologic conditions of sufficient severity as to affect cognitive functioning; (9) the presence of abnormalities from blood testing which were considered sufficient in magnitude to affect cognition or functioning; the blood testing included (a) complete blood counts, (b) a comprehensive metabolic panel, (c) serum B12 and folate levels, (d) thyroid function tests, specifically thyroxine (T4), triiodothyronine (T3), and thyroid stimulating hormone levels, and (e) screening for syphilis. Additionally, subjects were excluded from participation in this longitudinal study if they were receiving medications that might significantly affect cognitive functioning. If exclusionary factors were uncovered, then potential subjects were referred for treatment by community physicians. Eligible subjects were accepted for entry into our longitudinally followed research population.

Although subjects with complaints of cognitive decline frequently contacted our center for assistance, persons free of subjective or objective cognitive decline were relatively difficult to recruit for the study. Therefore, spouses and other community-residing persons were contacted to assist with our recruitment of this cohort.

Subject recruitment occurred from January 1, 1984, until December 31, 1997. During this period, 60 no cognitive decline (NCD) subjects were recruited. These subjects were subsequently followed over approximately 2-year intervals until December 31, 2001. All follow-up evaluations were completed without reference to the prior results.

Assessment measures in the present investigation included demographic variables, MMSE scores [14], Brief Cognitive Rating Scale (BCRS), axis 1-5 scores, and total BCRS axis 1-5 scores [15], Hamilton Depression Scale [13], total and item scores, and a psychometric test battery which has been in use at the NYU Alzheimer's Disease Center for several decades [2]. The specific tests used in this test battery are assessments of Paragraph Recall, Initial and Delayed Recall [16], Paired Associates Recall, Initial, and Delayed Recall [16], Memory for Designs [16], Digit Span Recall, Forwards [17], Digit Span Recall, Reverse [17], the Digit Symbol Substitution Test [17], and the WAIS-R Vocabulary subtest [17].

A combinatorial psychometric deterioration score (PDS) was also computed. This was derived from an equal weighing of the 9 tests described above in the test battery. Although the formula for this computation has been used previously (e.g., see [7]), the formula appears to not have been published previously. Therefore, we are describing the formula below:

$$
\begin{aligned}
& \mathrm{PDS}=(8-(((\mathrm{PARI})+(\mathrm{PARD})) / 45+((\mathrm{PRDI})+ \\
& (\mathrm{PRDD})) / 20+((\mathrm{DESN}) / 10)+((\mathrm{WASV}) / 80)+ \\
& ((\mathrm{DSST}) / 75)+((\mathrm{WASDIGF})+(\mathrm{WASDIGB})) / 17) \times 1.6)
\end{aligned}
$$

Wherein "PARI" is Paragraphs Initial Recall, "PARD" is Paragraphs Delayed Recall, "PRDI" is Paired Associates Initial Recall, "PRDD" is Paired Associates Delayed Recall, "DESN" is Design Recall of abstract shapes, "WASV" is the language and vocabulary measure from the WAIS, the "DSST" is the Digit Symbol Substitution Test from the WAIS, "WASDIGF" is the longest consecutive sequence of numbers recalled correctly, and "WAISDIGB" is the longest number sequence correctly recalled in the reverse order.
Reisberg et al. 


\section{Results}

As described previously [7], at baseline, there were 60 subjects in the NCD (GDS stage 1) subject group. Fortyseven of the 60 subjects with NCD at baseline were followed. Their mean baseline age was $64.1 \pm 8.9$ years; 26 subjects were females and 21 were males. The mean level of education was $16.1 \pm 2.4$ years. The mean baseline MMSE score was $29.6 \pm 0.8$. The subjects' last GDS staging assessment prior to 2002 was used to determine outcome. On this basis, subjects were divided into 2 groups: (A) decliners, if the final GDS rating was $\geq 2$, and (B) nondecliners, if the final GDS rating was stage 1. Thirty-six subjects were found to have declined and 11 subjects were nondecliners.

The future decliners were older at baseline $(p=0.008)$. There were no significant baseline between-group differences in gender, education, MMSE scores, BCRS total or individual axis scores, Hamilton Depression Scale total or item scores, or BEHAVE-AD total or item scores [18].

After controlling for age, gender, education, and follow-up time, there was a significant between-group difference in decline rate $(p<0.001)$. Also, after controlling for age, gender, years of education, and follow-up time, the combinatorial PDS was significantly lower at baseline in the future decliners $(p=0.035)$. Of the 9 psychometric variables included in the study, after controlling for age, gender, education, and follow-up time, future decliners had significantly lower baseline scores on PARI $(p=$ 0.036), from the Guild Memory Scale [16], Digit Span Recall, Forwards $(p=0.016)$, and Digit Span Recall, Backwards $(p=0.049)$, from the WAIS [17].

\section{Discussion}

This appears to be the first study to directly link PCD to the subsequent SCD, and by extension, to the MCI and to the dementia of the Alzheimer's type continuum (see Reisberg et al. [19] for a recent discussion of this continuum). A question which immediately arises from our present findings is when in the course of the human lifespan does this continuum of psychometric and subjective cognitive decline begin? At least one publication appears to have recently addressed some aspects of this question. Singh-Manoux et al. [20] conducted a prospective cohort study in which they investigated 10-year decline in cognitive function from a longitudinal dataset. Subjects were employees of civil service departments in London, UK. Study participants included 5,198 men and 2,192 women

Psychometric Cognitive Decline who were aged 45-70 years at the time of the initiation of cognitive testing. The investigators concluded that " $\operatorname{cog}$ nitive decline is already present in middle-age (age 4549)" [20].

However, a severe limitation of the study of Singh-Manoux et al. [20] is the complete absence of medical, psychiatric, neurologic, neuroradiologic, or clinical laboratory evaluations. Without the exclusion of other conditions, it is unclear how many of Singh-Manoux et al.'s [20] findings with respect to cognitive decline and aging should be attributed to $\mathrm{AD}$. Conversely, the present investigation specifically excluded conditions apart from incipient $\mathrm{AD}$ which could be attributed to the observed changes.

Since AD is known to be associated with age [21,22], removing age from the presentation of our findings by "controlling for age" may not be appropriate in terms of conveying the true nature and import of our observations. Accordingly, we are now presenting our data in this discussion without controlling for age.

It should be noted that there were no significant differences between our subject groups in gender, educational background, MMSE scores, BCRS axis 1-5 total scores, Hamilton Depression Scale total scores, BEHAVE-AD total scores, follow-up times, or follow-up visits.

In terms of the psychometric tests scores, there were very significant differences between the future nondecliner and the future decliner subjects. The combinatorial PDS, in which a lower score signifies better performance, was a mean of $1.13 \pm 0.90$ in the future nondecliners and $2.03 \pm 0.90$ in the future decliners $(p=0.008)$. Three of the 9 individual scores, in which a higher score signifies better performance, also showed significant differences, all in favor of the future nondecliner subject group. Specifically, on the Paragraph Initial Recall assessments, the subjects in the future nondecliner group scored a mean of $9.77 \pm 1.94$ at baseline, and the future decliner subjects scored $7.53 \pm 2.58$ at baseline. This difference was highly significant $(p=0.005)$. Also, the baseline Digit Symbol Substitution Test score mean in the future nondecliners was $61.36 \pm 7.50$, which was significantly higher than the scores in the future decliner subject group, i.e., $53.57 \pm 11.39(p=0.025)$. Finally, the baseline WAIS Digits Forward assessment was significantly higher in the future nondecliners, i.e., a mean of $7.82 \pm 0.98$, than in the future decliners, i.e., $6.64 \pm 1.45(p=0.009)$.

Hence, we believe this additional analysis, which did not control for age, in these subjects who were selected to be healthy and free of subjective or objective cognitive 
impairments at baseline lends further support for a process of cognitive decline for which we suggest the terminology "psychometric cognitive decline," which antedates the subsequent SCD and MCI stages of the eventual dementia of $\mathrm{AD}$.

\section{Acknowledgment}

The authors wish to acknowledge the contributions of our deceased colleagues, Dr. Steven Ferris and Dr. Emile Franssen, to the studies described in this publication.

\section{Statement of Ethics}

The subjects in this study had "no cognitive decline" at baseline and consented to this longitudinal study. All subjects were fully capable of providing consent. This longitudinal study was approved from the time of inception until the present time by the Institutional Review Board of NYU Langone Medical Center.

\section{Disclosure Statement}

Dr. Barry Reisberg, Dr. Yongzhao Shao, Dr. Sunnie Kenowsky, Dr. Karyn Marsh, Ms. Jia Bao, Dr. Carol Torossian, Mr. Alok Vedvyas, Mr. Gaurav Vedvyas, Dr. Thet Oo, Dr. Arjun Masurkar, and Dr. Thomas Wisniewski are employees of NYU Langone Health. Dr. Mesum Moosavi, Dr. Maja Buj, and Dr. Fauzia Arain were nontraditional volunteers at NYU Langone Health. Dr. Alan Kluger is presently an employee of Lehman College of the City University of New York and previously had been a full-time employee of the NYU School of Medicine. Dr. Fawad Malik is an employee of the Department of Pharmacy of Bellevue Hospital, New York, NY, USA.

\section{Funding Sources}

\section{Author Contributions}

Barry Reisberg, MD, conceived of the study, obtained funding for the study and provided oversight and direction for all aspects of the study, including the study analyses and the preparation of this manuscript. Dr. Reisberg worked closely on this study with Dr. Steven Ferris, who died in 2017. Yongzhao Shao, PhD, had primary responsibility for the statistical analysis of this study. Mesum Moosavi, MD, worked closely with Dr. Reisberg on the preparation of the manuscript for this study. Dr. Sunnie Kenowsky worked with Dr. Reisberg on the maintenance of the longitudinal study population for this study. Mr. Alok Vedvyas had primary responsibility for the maintenance of the database for the study, including data checking and associated data analyses including the analyses for the present report. Karyn Marsh, $\mathrm{PhD}$, supervised and provided oversight for the study personnel, helping to ensure proper follow-up of subjects and the accuracy of the study data. Ms. Jia Bao performed most of the statistical analyses for this study. Maja Buj, MD, assisted Dr. Reisberg with the preparation of the manuscript for this study. Carol Torossian, $\mathrm{PhD}$, assisted with the statistical analysis of this study. Alan Kluger, $\mathrm{PhD}$, who was previously an employee of NYU Langone Health, assisted with the recruitment of subjects for this study. Mr. Gaurav Vedvyas assisted in the maintenance of the database for this longitudinal study and with the associated analyses. Thet $\mathrm{Oo}, \mathrm{MD}$, assisted with recruitment of the subjects and maintained a follow-up subject schedule for this longitudinal study. Fawad Malik, MD, and Fauzia Arain, MD, assisted with the preparation of this report. Arjun V. Masurkar, MD, who is presently the Director of the Clinical Core of the NYU Alzheimer's Disease Center, and Thomas Wisniewski, MD, who is presently Director of the NYU Alzheimer's Disease Center, assisted in providing oversight for many aspects of the study and in the preparation of this report. All authors approved the final version of this report.

Published in Celebration of the 30th Anniversary of the inception of Dementia and Geriatric Cognitive Disorders 1990-2020.
This longitudinal study was supported in part by United States Department of Health and Human Services (DHHS) grants P30 AG08051 and AG03051 from the National Institute on Aging (NIA), by grant MH43486 from the National Institute of Mental Health of the US National Institutes of Health (NIH), by grant NCRRM01 RR00096 from the General Clinical Research Center Program and by Clinical and Translational Science Institute grant 1UL1RR029893 from the National Center for Research Resources of the NIH, by grant AG12101 from the NIA of the $\mathrm{NIH}$, by grants MH35976 and MH40410 from the National Institute of Mental Health of the NIH, by grants from the Stringer Foundation, Mr. William Silberstein, the Leonard Litwin Fund for Alzheimer's Disease Research, the Woodbourne Foundation, Mr. Zachary Fisher and Mrs. Elizabeth M. Fisher, the Fisher Center for Alzheimer's Research Foundation, the Estate of Rosalind Cherry, the Hagedorn Fund, and the Louis J. and June E. Kay Foundation.

\section{References}

20
Dement Geriatr Cogn Disord 2020;49:16-21 DOI: $10.1159 / 000507286$
1 Reisberg B, Ferris SH, de Leon MJ, Crook T. The Global Deterioration Scale for assessment of primary degenerative dementia. Am J Psychiatry. 1982 Sep;139(9):1136-9.

2 Reisberg B, Ferris SH, de Leon MJ, Sinaiko E, Franssen E, Kluger A, et al. Stage-specific behavioral, cognitive, and in vivo changes in community residing subjects with age-associated memory impairment and primary degenerative dementia of the Alzheimer type. Drug Dev Res. 1988;15(2-3):101-14.

3 Reisberg B. Dementia: a systematic approach to identifying reversible causes. Geriatrics. 1986 Apr;41(4):30-46.

4 Prichep LS, John ER, Ferris SH, Rausch L, Fang Z, Cancro R, et al. Prediction of longitudinal cognitive decline in normal elderly with subjective complaints using electrophysiological imaging. Neurobiol Aging. 2006 Mar; 27(3):471-81.
Reisberg et al. 
5 Reisberg B, Gauthier S. Current evidence for subjective cognitive impairment (SCI) as the pre-mild cognitive impairment (MCI) stage of subsequently manifest Alzheimer's disease. Int Psychogeriatr. 2008 Feb;20(1):1-16.

6 Mitchell AJ, Beaumont H, Ferguson D, Yadegarfar M, Stubbs B. Risk of dementia and mild cognitive impairment in older people with subjective memory complaints: meta-analysis. Acta Psychiatr Scand. 2014 Dec;130(6): 439-51.

7 Reisberg B, Shulman MB, Torossian C, Leng $\mathrm{L}$, Zhu W. Outcome over seven years of healthy adults with and without subjective cognitive impairment. Alzheimers Dement. 2010 Jan;6(1):11-24.

8 Jessen F, Amariglio RE, van Boxtel M, Breteler M, Ceccaldi M, Chételat G, et al.; Subjective Cognitive Decline Initiative (SCD-I) Working Group. A conceptual framework for research on subjective cognitive decline in preclinical Alzheimer's disease. Alzheimers Dement. 2014 Nov; 10(6):844-52.

9 Rabin LA, Smart CM, Crane PK, Amariglio RE, Berman LM, Boada M, et al. Subjective Cognitive Decline in older adults: an overview of self-report measures used across 19 international research studies. J Alzheimers Dis. 2015 Sep;48(s1 Suppl 1):S63-86.
10 Molinuevo JL, Rabin LA, Amariglio R, Buckley R, Dubois B, Ellis KA, et al.; Subjective Cognitive Decline Initiative (SCD-I) Working Group. Implementation of subjective cognitive decline criteria in research studies. Alzheimers Dement. 2017 Mar;13(3):296-311.

11 Slot RE, Sikkes SA, Berkhof J, Brodaty H, Buckley R, Cavedo E, et al.; Alzheimer's Disease Neuroimaging Initiative; DESCRIPA working group; INSIGHT-preAD study group; SCD-I working group. Subjective cognitive decline and rates of incident Alzheimer's disease and non-Alzheimer's disease dementia. Alzheimers Dement. 2019 Mar; 15(3):465-76

12 Rosen WG, Terry RD, Fuld PA, Katzman R, Peck A. Pathological verification of ischemic score in differentiation of dementias. Ann Neurol. 1980 May;7(5):486-8.

13 Hamilton M. A rating scale for depression. J Neurol Neurosurg Psychiatry. 1960 Feb; 23(1):56-62.

14 Folstein MF, Folstein SE, McHugh PR. "Minimental state". A practical method for grading the cognitive state of patients for the clinician. J Psychiatr Res. 1975 Nov;12(3):189-98.

15 Reisberg B, Schneck MK, Ferris SH, Schwartz GE, de Leon MJ. The Brief Cognitive Rating Scale (BCRS): findings in primary degenerative dementia (PDD). Psychopharmacol Bull. 1983;19:47-50.
16 Gilbert JG, Levee RF. Patterns of declining memory. J Gerontol. 1971 Jan;26(1):70-5.

17 Wechsler DA. The Measurement and Appraisal of Adult Intelligence. Baltimore (MD): Williams \& Wilkins; 1958. https://doi. org/10.1037/11167-000.

18 Reisberg B, Borenstein J, Salob SP, Ferris SH, Franssen E, Georgotas A. Behavioral symptoms in Alzheimer's disease: phenomenology and treatment. J Clin Psychiatry. 1987 May; 48(5 Suppl):9-15.

19 Reisberg B, Franssen E, Souren LE, Kenowksy $\mathrm{S}$, Janjua K, Veigne S, et al. Alzheimer's disease. In: Moroz A, Flanagan SR, Zaretsky H, editors. Medical Aspects of Disability. 5th ed. New York: Springer; 2017. pp. 31-90.

20 Singh-Manoux A, Kivimaki M, Glymour MM, Elbaz A, Berr C, Ebmeier KP, et al. Timing of onset of cognitive decline: results from Whitehall II prospective cohort study. BMJ. 2012 Jan;344:d7622.

21 Hebert LE, Scherr PA, Beckett LA, Albert MS, Pilgrim DM, Chown MJ, et al. Age-specific incidence of Alzheimer's disease in a community population. JAMA. 1995 May;273(17): 1354-9.

22 Zhang MY, Katzman R, Salmon D, Jin H, Cai GJ, Wang ZY, et al. The prevalence of dementia and Alzheimer's disease in Shanghai, China: impact of age, gender, and education. Ann Neurol. 1990 Apr;27(4):428-37. 